\title{
L'émergence des mythologies individuelles, du brut au contemporain
}

\author{
Magali Nachtergael \\ «Légion est mon nom, car nous sommes \\ beaucoup. » (Livre de Marc, 5 : 9)
}

\section{Introduction : le mythe individuel, un et multiple}

La notion de mythe individuel, ou personnel, peut être considérée de multiples points de vue: psychanalytique, esthétique, culturel, anthropologique, médiatique, voire ésotérique. L'émergence de la notion dans les années 1960 dans plusieurs domaines disciplinaires témoigne d'un processus d'individualisation du mythe. Dans le même temps, on le perçoit comme un élément central au sein de la représentation imaginaire des singularités et des identités. L'essor de l'anthropologie structurale dans les sciences humaines n'est évidemment pas étranger à cet intérêt critique grandissant pour les multiples arborescences mythologiques au sein des sociétés. Par un effet de contamination réciproque, le rêve d'une architecture sociale fondée sur le mythe s'applique de loin en proche à la structure psychique de l'individu, au point que l'on se met à imaginer qu'il formerait en lui son petit monde et ses petites mythologies, parfois jusqu'à la folie et au repli total sur soi. Avant de rentrer dans l'histoire des mythologies individuelles contemporaines et de tenter de comprendre comment le mythe personnel a participé à la fois à une grande révolution identitaire et esthétique au $\mathrm{XX}^{\mathrm{e}}$ siècle, il convient de revenir rapidement sur ce que la notion recouvre tout en esquissant ses contours théoriques. Esquisser, car il est loin d'être acquis que tous les auteurs ayant manié ces expressions de mythe personnel ou mythologie individuelle leur aient donné exactement la même signification. Aujourd'hui encore, le sens que ces mots, presque tombés dans l'usage commun, peuvent recouvrir ne correspond pas historiquement à une seule et même définition. Nous pouvons donc garder à l'esprit que, et cette caractéristique est commandée par sa nature même, de multiples visions du mythe 
personnel cohabitent à une même période. D'hier à aujourd'hui, le sens s'est encore déplacé et continue à chasser devant lui dans un contexte marqué par l'environnement numérique et l'hyperconnectivité. Nonobstant ces variations, sa prégnance et sa permanence en font une réalité à la fois discursive et épistémologique dont l'impact sur les productions esthétiques dans la seconde moitié du $\mathrm{XX}^{\mathrm{e}}$ siècle fut déterminant.

Dans les années 1960, sous l'influence des humanités classiques, le terme de mythe reste encore très fortement lié aux récits antiques et fondateurs de la civilisation moderne, violemment allégoriques, moralistes et logothétiques. La première destitution de cette forme du mythe, jeté de son piédestal antique, fut l'opération de mise à distance effectuée par l'anthropologue Claude Lévi-Strauss. Son opération critique fait d'emblée entrer le mythe dans la fabrique d'une fiction sociale structurante au plus proche de l'individu, puisqu'il s'intéresse à la vie de famille d'abord chez les Indiens Nambikwara (1948), puis à travers les «structures élémentaires de la parenté » (1949) ${ }^{1}$. Lévi-Strauss sait toutefois qu'il propose un miroir révélateur de la société occidentale, structurée selon lui à partir d'un modèle molaire de parenté unique et élargi. Dans "Linguistique et anthropologie " en 1952, Lévi-Strauss corrèle parenté et mythologie, scellant au cœur même de l'espace familial le maillon du mythe ${ }^{2}$. Il inclut à ce maillage les rites magiques et les croyances collectives rencontrés au quotidien dans ces sociétés éloignées et tend à l'homme occidental un tableau de ses propres mœurs, tout à coup dépouillé de ses artifices modernes et scientifiques qui lui donnaient l'illusion d'un univers sécularisé et rationnel ${ }^{3}$.

La mise en perspective de ces deux univers les ramène en fait dos à dos. Lévi-Strauss invoque par exemple une forme de poésie incantatoire à l'usage des chamans d'Amérique du Sud. Ces poèmes ont pour fonction, tel un vade-mecum, d'aider les femmes à accoucher ou à soigner un malade : Lévi-Strauss compare ces textes poétiques de références à l'usage des mythes dans la cure psychanalytique freudienne $e^{4}$. Toutes ces hypothèses anthropologiques sont suivies de

${ }^{1}$ Claude Lévi-Strauss, La Vie familiale et sociale des Indiens Nambikwara, Société des américanistes, 1948, et Les Structures élémentaires de la parenté, PUF, 1949.

${ }^{2}$ Claude Lévi-Strauss, Anthropologie structurale, Plon, 1958, p. 86-88.

${ }^{3}$ Voir Claude Lévi-Strauss, La Pensée sauvage, Plon, 1962, et plus particulièrement la notion de bricolage mytho-poétique mise en perspective avec les pratiques de l'art brut et notamment le palais entièrement bricolé du Facteur Cheval, p. 26.

${ }^{4}$ Ibid., p. 223 : «On a vu que la seule différence entre les deux méthodes, [...] concernerait l'origine du mythe, retrouvé, dans un cas, comme un trésor individuel, et reçu, dans l'autre, de la tradition collective. [...] Ce qu'il convient de se demander, c'est si [...] le pouvoir traumatisant de ces situations ne provient pas du fait qu'au moment où elles se présentent, le sujet les expérimente 
très près par Roland Barthes qui publie à son tour ses Mythologies en 1957, recueil d'articles dans lequel le sociologue alors en poste au CNRS démonte minutieusement les constructions idéologiques petites-bourgeoises de la société moderne. Mais Barthes, s'il est structuraliste dans sa méthode, ajoute un nouveau matériau au support du mythe : l'image, que Lévi-Strauss semble minorer dans son étude, préférant s'appuyer de façon plus affirmée sur les sciences du langage. Avec Barthes, le territoire du mythe s'élargit donc aux médias qui lui sont contemporains, le cinéma, la presse, mais aussi les expositions ${ }^{5}$. Le chemin du mythe dans les années 1960 paraît bifurquer ici une première fois, d'un côté dans le giron du rite magique, de l'autre dans l'espace public des médias. La disjonction n'est toutefois qu'apparente: le mythe et sa force rituelle se déplacent, et Barthes transpose très judicieusement les théories anthropologiques de Lévi-Strauss au cœur de la modernité occidentale, réaffirmant le caractère culturel de ces grands récits de société. Point de poèmes chamans pour aider les femmes à Paris certes, mais des magazines comme Elle ou Match où abondent des récits et courriers du cœur censés faire «rêver la secrétaire et la bourgeoise ", comme le confient les journalistes de Match au sociologue Luc Boltanski6. Derrière l'entreprise de déconstruction mythologique de Lévi-Strauss et Barthes, on retrouve aussi la préoccupation très marxiste d'un dessillement de la population face à la société du spectacle (Guy Debord, 1967) et l'illusion fictionnelle (L'Ère du soupçon de Nathalie Sarraute, 1956), fers de lance de la propagande et de l'idéologie bourgeoises. Ces réactions épidermiques face à tout ce qui peut s'apparenter de près ou de loin à un endoctrinement par la croyance sont donc combattues avec virulence, sous la férule d'un doute généralisé. La recherche résolue et rigoureuse de la vérité s'accomplit soit par un retour au réalisme historique et au démontage mythologique, soit par une réévaluation de formes d'expression brutes ou sauvages, c'est-à-dire non encore

immédiatement sous forme de mythe vécu. » On peut faire intervenir ici le livre de Mircea Eliade, Aspects du mythe, paru en 1963, et Le Sacré et le Profane, en 1965.

5 L'édition illustrée de Mythologies par Jacqueline Guittard a rendu manifeste l'ancrage médiatique de ces mythes modernes, Seuil, 2010. L'exposition "mythologique » est The Family of Man, organisée par Edward Steichen en 1955 au MoMA de New York et qui fit une tournée en Europe, archétypale dans la formation d'un mythe de société unie, mythe qui s'appuie de façon ostentatoire sur une parentèle construite en images.

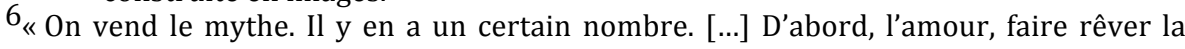
secrétaire et la bourgeoise con [...]», Luc Boltanski, "La Rhétorique de la figure », in Pierre Bourdieu (dir.), Un art moyen. Essais sur les usages sociaux de la photographie, «Le Sens commun », Les Éditions de Minuit, 1965, p. 193. 
perverties par cette société spectaculaire-marchande génératrice de ses propres mythographies.

\section{Mythe et psychanalyse : le repli intérieur}

Le mythe individuel apparaît également comme l'espace d'un repli face à une culture de masse qui se caractérise par une standardisation massive des foyers et des lieux de l'intimité. Les formes artistiques qui procèdent de cette domination de la marchandise et des objets sur l'individu sont évidemment incarnées par le pop art. Conscients de l'appropriation à double tranchant des objets de consommation par les artistes américains, les nouveaux réalistes français, dans un même élan que Jean Dubuffet, tentent de contrer cette adhésion au consumérisme artistique. Dubuffet choisit une position extrême et risquée en se rangeant du côté des artistes marginaux, minorés et populaires à travers la création en 1947, avec le soutien d'André Breton, de la Compagnie de l'Art Brut. Là encore, l'acception du mythe et du mythe individuel revêt une tout autre coloration que chez Lévi-Strauss et Barthes. Depuis Le Paysan de Paris et les années 1920, une "mythologie moderne", essentiellement urbaine, est à l'œuvre chez les surréalistes. Si elle puise sa source dans la formation psychiatrique des membres fondateurs du groupe et leur lecture assidue de Freud, si elle affiche dans Le Manifeste du surréalisme la recherche d'une vérité psychique brute qui marauderait aux portes de la folie, elle ne se départit pas tout à fait de ce qui constitue les linéaments des mythologies barthésiennes. Nadja pose tous les éléments de cette mythologie hybride, entre représentations médiatiques populaires et exploration individuelle. On se plonge dans le décor urbain des années folles, son cinéma populaire, son théâtre de boulevard, ses marchés aux puces, ses voyantes et médiums, on rencontre la jeune héroïnomane Nadja, dessinatrice éclairée, mais qui finit internée dans un asile à Bailleul, non loin de Lille, et surtout on visite le panorama mental de l'auteur du livre, André Breton, en suivant une galerie photographique. En guise d'incipit de l'ouvrage, il pose sans détour la véritable question matricielle de l'ouvrage et le sujet principal du livre : «Qui suis-je ? » À ce titre, il faut voir en Nadja la première expression d'une mythologie individuelle qui établit dans un entremêlement subtil la mythologie du surréalisme même. N'a-t-on pas qualifié Breton de "pape» du surréalisme? La pauvre Léona Delcourt fut malheureusement plus une muse déchue, victime sacrifiée sur l'autel du grand plan surréaliste, qu'une héroïne de roman, et sa triste fin, ses appels au secours répétés à Breton, n'ont dû qu'augmenter sa culpabilité (lui, l'ancien assistant du professeur Babinski, élève de Charcot, n'avait rien vu) et le pousser à sublimer cet échec en un récit 
qui a en effet fondé une mythologie nouvelle, mais bien aux dépens de celle que Breton qualifiait de "génie libre ».

On peut toutefois reconnaître à Breton, et cela dès 1928, son engagement pour un art autre, singulier. Les dessins de Nadja font en effet partie de ces premières œuvres brutes qui ont acquis un statut véritablement artistique et légitime, et la collaboration de Dubuffet avec Breton s'entend aussi comme un accord de fond sur le renversement des valeurs esthétiques académiques et institutionnelles. L'influence de la psychanalyse participe pour beaucoup de leur choix d'exposer des œuvres brutes de créateurs retenus dans des institutions psychiatriques. Bien entendu, l'idée d'un «art des fous » n'est pas une invention des années 1950, elle était déjà à l'étude dans les années 1920 et Breton en avait eu connaissance par les médecins Walter Morgenthaler et Hans Prinzhorn qui publient leurs ouvrages pionniers sur la production artistique des aliénés à partir de 1921. Et ils travaillent depuis 1908 à ces œuvres jusque-là utilisées uniquement à des fins diagnostiques ${ }^{7}$. La concomitance entre ces travaux dans des asiles et l'apparition des avant-gardes à Paris (fauvisme, expressionnisme, cubisme, primitivisme) témoigne là encore d'une réévaluation générale des valeurs et des formes créatives, ouvrant la voie à de nouvelles expérimentations, mais aussi à un renouvellement des regards sur ce qui fait œuvre.

Pour cela, il faut reconnaître que les surréalistes sont parvenus à faire entrer de plain-pied la question psychanalytique et l'expression de la psyché, que ce soit par la folie ou les rêves, dans le champ des arts. La volonté de fonder un "mythe nouveau ${ }^{8}$ » ou de réactiver la dimension mythique de certains objets, comme les fétiches, n'est jamais loin. Par cette brèche, Georges Bataille, dès 1929 avec la revue Documents, étend son programme artistique à l'archéologie, l'ethnologie et la philosophie: tout le matériau brut recueilli et reproduit en photographie dans la revue prolonge et approfondit la démarche documentaire initiée dans La Révolution surréaliste, mais en mettant l'accent sur une quête méthodique de vérité originaire et spirituelle. Ethnologie, psychanalyse, art et documents bruts se croisent à un moment charnière de l'histoire, avant l'effondrement définitif des grands mythes de civilisation ${ }^{9}$. Ils vont se rematérialiser

${ }^{7}$ Walter Morgenthaler, Ein Geisteskranker als Künstler, Berne-Leipzig, Bircher, 1921, et Hans Prinzhorn, Bildnerei der Geisteskranken (Expressions de la folie), Berlin, Springer Verlag, 1922.

${ }^{8}$ Dans une dédicace d'Arcane 17, Breton avait signé : « Et vive alors le mythe nouveau !», cité par Michel Beaujour, "André Breton mythographe: Arcane 17», Études françaises, $n^{\circ} 2$, vol. 3, 1967, p. 216.

${ }^{9} \mathrm{Si}$ Jean-François Lyotard prend acte en 1979 dans La Condition postmoderne de la « fin des grands récits», il s'agit d'un processus qui s'ancre vraisemblablement dans 
après la seconde guerre mondiale dans un tout autre contexte philosophique et social, et intégrer progressivement les bouleversements de cette ère nouvelle qui s'ouvre en 1945, ère marquée par le doute et la tentative de définition de l'existence après l'expérience annihilante de la guerre totale et du traumatisme de l'Holocauste. Le souvenir d'un art dégénéré et de l'eugénisme répugnant finit de convaincre de la nécessité absolue de revenir à cette autre conception de l'expression individuelle rejetée par l'occupant nazi, et, ainsi, à une autre vérité, sous le signe de la liberté retrouvée. Si les gueules étaient cassées après 1917, les esprits furent défigurés après 1945. L'expressionnisme abstrait, grand mouvement de l'après-guerre aux États-Unis, ou l'abstraction lyrique et le tachisme en France, avec Wols, Jean Fautrier, Antoni Tapiès et bien entendu Jean Dubuffet, témoignent de cette impossibilité de représenter la réalité du monde d'avant. En 1947, Breton, aidé de Duchamp, inaugure l'exposition internationale surréaliste autour du thème imposé "Un nouveau mythe collectif», tout juste un an avant la création de la Compagnie de l'Art Brut ${ }^{10}$. Il n'est donc pas étonnant dans ce contexte de reconstruction symbolique que les regards se tournent vers des personnes aussi protégées des tourments du monde tout en révélant une part de sa vérité immaculée. La constitution de la Compagnie de l'Art Brut s'inscrit dans ce contexte historique qui fait le pont entre des positions esthétiques énoncées avant la guerre et les préoccupations existentielles d'une nouvelle génération d'artistes. Cette dernière s'empare alors de ces outils esthétiques, scientifiques et philosophiques pour opérer un déplacement radical des valeurs de l'art et de ses mythes.

À l'orée des années 1960, le changement de génération est manifeste et les effets du rationalisme d'après-guerre se font sentir. L'essor des sciences humaines imprègne petit à petit la production artistique et l'on voit apparaître un art conceptuel qui travaille la question du langage, à l'instar des structuralistes, à partir de textes, documents et archives. Dans le même temps, en 1963, apparaît en littérature la notion de "psychocritique» par l'entremise de l'ouvrage de Charles Mauron, Des métaphores obsédantes au mythe personnel. Introduction à la psychocritique. À cette époque, la célèbre conférence de Jacques Lacan, «Le Mythe individuel du névrosé », a été prononcée depuis plus de dix ans au Collège philosophique

l'après-guerre pour atteindre son apogée dans les années 1970, et l'émergence des mythologies individuelles dans ce mouvement de pulvérisation des grands récits n'est pas étrangère à l'identification de ce phénomène global de détérioration mythologique.

${ }^{10}$ André Breton, Benjamin Péret, Victor Brauner, Henry Miller et al., Exposition internationale du surréalisme. Le Surréalisme en 1947, Pierre à feu - Maeght, 1947. 
(1952). Lacan ne cache d'ailleurs pas sa dette à l'anthropologie structurale et admet que "ces intérêts se sont nourris et élargis de bien des choses [...] apprises de Claude Lévi-Strauss ${ }^{11}$ ». Pour décrire les ressorts de la névrose, Lacan convoque notamment la notion de " mythe familial ${ }^{12}$ », tout en lui ajoutant le caractère obsessionnel de cette mythologie intime qui accompagne généralement un dédoublement narcissique. Toute cette nomenclature réapparaît dans le discours critique des années 1970 chez des critiques, auteurs, artistes ou commissaires d'exposition baignés de psychanalyse et férus d'ethnologie. Ce discours, porté depuis longtemps dès les années 1920 par les surréalistes puis par le Collège de sociologie, fait partie de l'arrière-plan théorique dans lequel surgissent les œuvres issues des mythologies individuelles, mais aussi les productions d'art brut. Elles entretiennent donc en guise de décor discursif, en plus des nombreux points de contact formels, une relation critique qui se place dans le même univers de référence. L'intérêt pour le mythe personnel et sa fonction sociale se fraye des chemins profondément enracinés dans l'imaginaire collectif. Quand, d'un côté, l'art brut garde une part de mystère créatif et donne la sensation de toucher à une pépite d'or encore dans sa gangue unique, de l'autre, le récit personnel rétrospectif mis en place dans la cure psychanalytique s'élargit au point d'être identifié comme genre littéraire à part entière dans les années $1970^{13}$. Mis en perspective avec la démarche exploratoire des sciences humaines, art brut et expression narrative de soi participent au premier plan à l'étude de l'intériorité et de l'homme au sens large. Cette matière produite dans un cadre mythopoétique, qu'elle soit littéraire, technique ou plastique, laisserait donc voir les différentes faces d'un même dé, observé selon un point de vue tantôt analytique et tantôt esthétique.

\section{La mythologie individuelle: vraie trouvaille, faux concept ?}

C'est dans ce Zeitgeist bouillonnant et éclectique que débute la carrière du commissaire d'exposition Harald Szeemann (1933-2005), directeur de la Kunsthalle de Berne dès 1961, et qui rendra célèbre dans le monde de l'art l'expression «mythologies individuelles » en

\footnotetext{
11 Jacques Lacan, «Intervention après un exposé de Claude Lévi-Strauss à la Société française de philosophie, "Sur les rapports entre la mythologie et le rituel..." ", in Le mythe individuel du névrosé [1952], Champ freudien, Seuil, 2007, p. 101.

${ }^{12} \mathrm{Ce}$ « mythe familial » n'a pas été vécu par le sujet, il lui est transmis comme un récit qui peut devenir un élément névrotique. Dans le cas de "L'homme aux rats", Lacan identifie des éléments de ce mythe (des " mythèmes", selon l'expression de Claude Lévi-Strauss, ibid., p. 103) pour justifier les raisons qui ont poussé le père à épouser la mère, ibid., p. 22.

13 Philippe Lejeune, Le Pacte autobiographique, Poétique, Seuil, 1975.
} 
désignant ainsi toute une section de la $5^{\mathrm{e}}$ documenta de Cassel en $1972^{14}$. Mais Szeemann, en liant progressivement ces mythologies aux obsessions, induit une interprétation à dominante psychanalytique, au risque d'enfermer la notion dans une réception univoque. De plus, Szeemann n'est pas sensible de la même façon, en Suisse allemande, à la relecture du mythe par les structuralistes français, et particulièrement par Roland Barthes (que Szeemann a lu assidûment) qui s'attache surtout à décrire la société de son temps. Par comparaison, Lévi-Strauss adopte une position humaniste à même de couvrir un spectre humain large et diachronique, tandis que Barthes fait le choix de cibler un contexte contemporain, national (la France), médiatique (la presse illustrée) et social particulier (le monde petit-bourgeois). Ainsi, si l'acception de la mythologie moderne et individuelle varie en fonction des dominantes disciplinaires et des positions théoriques (Barthes est plus nettement marxiste), elle est aussi conditionnée par une réception culturelle marquée par des frontières linguistiques et nationales. La position légèrement excentrée de Harald Szeemann marque toutefois un véritable pivot dans les années 1960 entre la France et l'Allemagne. Jean-François Chevrier identifie très clairement ce hiatus culturel dont la compréhension de la notion de «mythologie » se fait un des révélateurs :

«[...] le modèle traditionnel du mythe comme représentation collective d'une société fondée sur le sacré, était largement transformé, voire dévalué, par cette nouvelle "mythologie" quotidienne, petite-bourgeoise, qui était suspectée, depuis les travaux de Roland Barthes, de produire une naturalisation antihistorique des phénomènes culturels. [...] Il faut aussi constater qu'elle n'a pas du tout pris la même signification selon les contextes culturels (et nationaux) dans lesquels elle s'est manifestée. Dans une même génération d'artistes nés autour de 1940 [...], la démarche des Français fut celle du doute et leur usage de la photographie venait d'une attraction pour une cause et un objet de ce doute ; les Allemands, au contraire, bénéficièrent de la présence de Beuys, et en amont, à travers lui, d'une tradition romantique toujours vivante, et c'est avec un humour plus "violent", ou en tout cas, une violence plus

${ }^{14}$ Voir le catalogue original: Marlis Grüterich et Harald Szeemann, documenta 5. Befragung der Realität, Bildwelten heute, cat. exp. du 30 juin au 8 octobre 1972, Neue Galerie, Schöne Aussicht, Museum Fridericanum, Friedrichplatz, Cassel, 1972. 
cadrée, [...] qu'ils furent amenés à s'exprimer avec la photographie $[\ldots]^{15}$ ».

Szeemann, à cheval entre deux cultures et deux langues, concilie habilement des productions dont l'orientation mythologique prend des chemins parfois divergents, ce qui fut justement l'objet de critiques à l'égard de ses expositions considérées souvent comme trop hétéroclites. Évidemment, certaines tendances semblent non seulement contradictoires mais incompatibles. D'un côté, Szeemann défend et présente des œuvres baignées de spiritualisme naturaliste et d'un individualisme postromantique typiquement germaniques. Cette tendance est incarnée par la grande figure mythographique de Joseph Beuys et son disciple, Lothar Baumgarten ${ }^{16}$. D'un autre côté, il met également en avant des œuvres plus directement tournées vers une mythologie de l'individu à l'ère des médias et de la facticité identitaire, une tendance que Christian Boltanski, Annette Messager et Jean Le Gac font vivre sur la scène parisienne des années post$1968^{17}$.

Harald Szeemann a façonné le visage artistique de la seconde partie du XX ${ }^{\mathrm{e}}$ siècle. S'étant d'abord fait positivement connaître pour sa première exposition majeure sur les Peintres-poètes / poètespeintres en 1957 (Saint-Gall, Suisse), il devient quatre ans plus tard directeur de la Kunsthalle de Berne, sa ville natale. C'est là qu'il réalise, entre autres, l'exposition révolutionnaire Quand les attitudes deviennent formes en 1969 sur l'art conceptuel, peu de temps après avoir exposé la collection d'art brut de Hans Prinzhorn ${ }^{18}$. Cette corrélation paratactique entre art brut et art conceptuel exposés dans une même programmation doit être interprétée dans la continuité de sa démarche. En 1972, Szeemann est en charge de la

15 Jean-François Chevrier, Photo-Kunst. Arbeiten aus 150 Jahren. Du XXe au XXIe siècle, aller et retour, cat. exp. Graphische Sammlung, 11 novembre 1989-14 janvier 1990, Staatsgalerie, Stuttgart, 1990, p. 71.

${ }^{16}$ Voir Joseph Mouton, "Remarques autour de la narration beuyssienne », in Jérôme Game, Le récit aujourd'hui, arts, littérature, Saint-Denis, Esthétiques hors cadre, Presses Universitaires de Vincennes, 2012, et Jean-Philippe Domecq, "Les mythes solitaires ", Communiquer / transmettre, Cahiers de médiologie, $\mathrm{n}^{\circ} 11$, vol. 1, 2011, p. 319-323.

${ }^{17}$ Voir Jean-Marc Poinsot, Une scène parisienne, 1968-1972. Christian Boltanski, Bernard Borgeaud, André Cadere, Paul-Armand Gette, Jean Le Gac, Annette Messager, Gina Pane, Sarkis, Archives de la critique d'art, Centre d'histoire de l'art contemporain, Rennes, 1991.

${ }^{18}$ L'exposition Live in Your Head. When Attitudes Become Forms a fait l'objet d'une reconstitution du 1er juin au 3 novembre 2013 à la Fondation Prada, Ca'Regina, Venise, sous le commissariat de Germano Celant, spécialiste de l'arte povera. En 1963, du 24 août au 15 septembre, il propose une exposition Bildnerei der Geisteskranken - Art brut - Insania Prigens, à la Kunsthalle de Berne. Szeemann reprend directement le titre de l'ouvrage de Prinzhorn pour cette exposition, puis pour une section de la documenta 5 . 
documenta 5 de Cassel: c'est à cette occasion qu'il présente les «Mythologies individuelles » sous la forme d'une grande section qui mêlait tout ensemble mouvements et artistes en apparence opposés, selon un principe déjà éprouvé pour Quand les attitudes deviennent formes. On identifie par exemple l'arte povera avec Mario Merz et Giuseppe Penone notamment, l'art conceptuel au sens large avec Walter de Maria, Dan Graham ou Marcel Broodthaers, Fluxus avec La Monte Young ou Ben Vautier, mais aussi des performances avec des artistes aussi divers que Yoko Ono, Vito Acconci, Rebecca Horn, Joseph Beuys ou Vettor Pisani ${ }^{19}$. Les artistes bruts, comme Adolf Wölfli ou Armand Schulthess, équivalent suisse du Facteur Cheval, y côtoient ces artistes établis. Malgré la diversité déroutante de cet assemblage, Szeemann s'impose comme une des figures majeures du monde artistique des années 1970, au point que la nouvelle figure de commissaire-auteur qu'il impose devient un symptôme de l'individualisation mythographique à tous les étages. En lançant le concept de "mythologie individuelle", Szeemann se trouve luimême emporté par ce renouveau de l'auteur, de l'artiste, mais aussi de l'individu, ce dont il a parfaitement conscience. Dépassant les clivages artistiques, Szeemann entre pleinement dans la culture du contemporain.

La suite de son parcours et ses nombreux écrits, entretiens, font pénétrer un peu plus loin dans cette fabrique artistique contemporaine de l'individu. Pour preuve, en 1974, soit deux ans après la documenta, il inaugure une exposition entièrement consacrée à son grand-père, Étienne Szeemann, coiffeur et «pionnier» anonyme ${ }^{20}$. C'est une fois encore à Berne, théâtre familial originel, qu'a lieu cette exposition mythographique qui présente les objets quotidiens d'un coiffeur comme des œuvres d'art. Ce retour aux traces de l'aïeul, modeste artisan et néanmoins créateur innovant dans son milieu, fait un pendant allégorique aux Demeures d'Étienne-Martin qui avaient tant fasciné Szeemann et lui avaient donné l'idée de «mythologie individuelle ». D’Étienne-Martin au grand-père Étienne en passant par la documenta, les expériences scénographiques de Szeemann le conduisent progressivement à l'idée d'un "Musée des obsessions " qui va supplanter la mythologie individuelle. C'est dans l'action muséale avant tout et par exclusion que le sens de la mythologie individuelle se précise. Étant entré dans le musée de ses propres obsessions, Szeemann assume alors pour

\footnotetext{
${ }^{19}$ Voir la réédition partielle du catalogue de la documenta: Tobia Bezzola et Roman Kurzmeyer (dir.), «documenta 5. Befragung der Realität. Bildwelten heute», in Harald Szeemann With By Through Because Towards Despite, Voldemeer, Zürich, 2007, p. 312-367.

${ }^{20}$ Harald Szeemann, Grossvater - ein Pionier wie Wir, 16 février-25 avril 1974, Galerie Toni Gerber, Berne.
} 
lui-même une critique qui lui avait été faite pour la documenta et admet enfin : " [je tenais] à me fabriquer mon propre mythe 21 ». Le concept de mythologie individuelle, lors de la documenta, n'a pas de lisibilité immédiate, en raison de la profusion des formes esthétiques et de la variété des démarches d'artistes ${ }^{22}$. Mais Szeemann le prolonge très rapidement vers l'exploration de l'intime et de la psyché jusqu'à trouver son aboutissement dans ce "Musée des obsessions », dont Szeemann déclare qu'il ne sépare la mythologie individuelle que d'un pas.

La démarche de Szeemann ne peut se réduire à un seul coup d'éclat, même si l'on aura fait remarquer à plusieurs reprises l'indigence théorique soutenant sa notion. Pour démystifier l'impact de cette expression ou pour s'en détacher lui-même, Christian Boltanski explique que le titre avait été choisi au dernier moment, sans réflexion préalable 23 . L'interview de Szeemann avec Gabriele Mackert en 2002 témoigne par ailleurs de la totale autonomie qu'a prise l'expression, comme si elle avait échappé à son créateur et vécu ultérieurement sa propre vie. À l'occasion d'une reconstitution des archives de la documenta 5 à la Kunsthalle de Vienne, le catalogue fait le point sur le contexte et les suites de l'événement, trente ans plus tard. Mackert signale à Szeemann que l'expression n'apparaît que dans le petit préambule au catalogue Befragung der Realität, par ailleurs peu fourni en textes critiques. Le commissaire assume la légèreté conceptuelle, au point d'en faire le mot d'ordre de la manifestation :

«G. Mackert: [...] Les «mythologies individuelles », la grande trouvaille, le slogan, qui a perduré comme un énoncé fondateur jusqu'à aujourd'hui, reste sans théorie, sans le moindre commentaire.

H. Szeemann: C'était alors cela l'idée: le lancer sans commentaire, tel quel. J'étais par-dessus tout intéressé par l'exposition, et pas par le fait d'écrire une préface ou un texte.

21 Harald Szeemann, « Agence pour le travail intellectuel à la demande au service de la vision d'un Musée des obsessions ", Écrire les expositions, trad. fr. Mariette Althaus, La Lettre volée, Bruxelles, 1996, p. 48.

22 «Avec une approche encyclopédique, il décide de présenter des objets qui n'appartiennent pas à la sphère artistique et crée un mélange d'objets communs et d'objets fétiches qui appartiennent à la tradition populaire, politique, kitsch, à l'art religieux ou encore à l'Art brut. Ainsi d5 peut être perçue comme un immense "cabinet de curiosités" », Lucia Pesapane, "documenta 5 : Enquête sur la réalité - Imageries d'aujourd'hui, $1972 »$, Florence Derieux (dir.), Harald Szeemann, Méthodologie individuelle, Le Magasin, Grenoble et JRP/Ringier, Zürich, 2007, p. 95.

${ }^{23}$ Entretien privé avec l'auteur, École des Beaux-Arts de Paris, Paris, 24 janvier 2008. 
J'ai ensuite donné des interviews et j'ai attiré l'attention sur cette subjectivation $[\ldots]^{24}$.»

Glissant de la "mythologie individuelle » à la subjectivité, Szeemann élude en fait la véritable question. Il poursuit d'ailleurs en relatant une anecdote qui trahit la désinvolture théorique qui avait accompagné une intuition pourtant géniale. Une partie de l'exposition devait accueillir Daniel Spoerri et proposer une performance qui ne put se faire, faute de finances (un lancer de tartes sponsorisé par Motta). L'espace vacant dut être converti en toute urgence et devint une série de Künstlermuseen (musées d'artistes) dans la Neue Galerie. Ce contretemps majeur laissa simplement moins de temps à Szeemann pour s'occuper de l'appareillage critique de cette monumentale exposition à la logistique pharaonique. Reprendre une expression déjà utilisée au sujet des sculptures d'Étienne-Martin était donc une solution de facilité, une désinvolture qui ne manqua pas de déplaire à nombre d'artistes, notamment américains, Robert Morris au premier chef ${ }^{25}$. Oscillant entre son «Musée des obsessions», «l'Agence pour le travail intellectuel » ou tout simplement le droit à la subjectivité, Szeemann tente malgré tout de donner à son idée un contour plus précis, à l'occasion d'un article paru dans Kunstnachrichten en novembre $1972^{26}$. Il y invite à considérer une acception large de la mythologie individuelle, celle-ci n'ayant pas de vocation didactique première. Il ne s'agit pas de proposer une lisibilité formelle, conceptuelle ou thématique. Szeemann revendique donc l'hétéroclisme et surtout la place centrale des artistes bruts, dans ce qu'ils peuvent apporter de pur et authentique dans la condition artistique contemporaine :

«Si les individualismes hors-schémas en avaient été exclus, elle aurait alors été touchée dans son "évènementialité", dans sa

24 Gabriele Mackert: [...] Die «Individuellen Mythologien», die grosse Erfindung, das Schlagwort, das als sprachliche Setzung bis heute überdauert, bleibt ohne Theorie, ohne jeglichen Kommentar. [...]

Harald Szeemann: «Das war es doch gerade : Es unkommentiert reinzuwerfen. Ich war vor allem an der Ausstellung interessiert und nicht daran, Vorworte oder Texte zu schreiben. Ich habe nachher Interviews gegeben und auf diese Subjektivierung hingewiesen", in Gabriele Mackert, Skandal and Mythos. Eine Befragung Harald Szeemans documenta 5 (1972), Vienne, Kunsthalle Wien Project Space, 2002, p. 19-20 (notre traduction).

25 Voir l'article à charge de l'artiste qui refusa de participer à la documenta, Robert Morris, "Regarding documenta V », Flash Art, mai-juin 1972. Robert Smithson, Carl Andre, Sol LeWitt, Donald Judd et d'autres s'insurgeaient notamment de voir leurs œuvres classées dans certaines catégories sans leur consentement.

${ }^{26}$ Cet article a été traduit et est publié sous le titre « Mythologies individuelles » dans le recueil quasiment introuvable, Harald Szeemann, Écrire les expositions, op. cit., p. 29-33. 
moelle. [...] les mythologies individuelles [...] préfigurent ce que fut l'objectif de chacune des expositions de ces dernières années : rendre perceptible une attitude exemplaire vécue en tant qu'individuum, et ainsi rendre visible l'anticipation d'identité qui, seule, devrait mettre en évidence et présenter une société meilleure, plus créative et plus consciente [...]. ${ }^{27}$ »

La mention de ces «individualismes hors-schémas» renvoie directement aux œuvres d'Adolf Wölfli que Szeemann fut un des premiers à exposer et à désigner comme procédant d'une mythologie individuelle ${ }^{28}$. Il avait intégré ses œuvres dans la section du même nom alors qu'une autre section, Bildnerei der Geisteskranken, en référence à Prinzhorn, accueillait plus spécifiquement l'art dit « des fous ». La perspective de Szeemann, heureuse, utopiste, se veut aussi la préfiguration d'une expression libre de l'individu au-delà du champ de l'art, ouvrant à " une société meilleure, plus créative et consciente ». Dans ce dernier point, il intègre clairement la réflexivité critique qui en fait un écho transformé du désir de rationalité scientifique promue par les structuralistes.

\section{La recherche de la singularité : formes vécues}

Fabien Faure a montré, dans son ouvrage sur Mario Merz et Étienne-Martin, que primitivisme et anthropologie représentaient une matrice théorique très importante chez Szeemann et qu'il avait été aussi l'artisan de ce rapprochement conceptuel dans les arts. Faure rappelle d'ailleurs qu'à l'origine le «pavillon des Mythologies individuelles devait même s'appeler Chamanisme et mystique sous l'influence de Beuys ${ }^{29}$ ». Ces informations orientent évidemment une perception contrastée du terme tel que Szeemann l'a manié, et tel que le public français a pu ensuite le percevoir. Si l'on se penche un peu plus encore sur les quelques commentaires que fait Szeemann de son propre concept, on remarque que son acception diffère sensiblement par exemple de celle que Catherine Millet lui confère en 1996 dans son ouvrage L'Art contemporain en France à propos de Christian Boltanski, Jean Le Gac, Annette Messager ou même Sarkis. On retrouve certes un mouvement de va-et-vient entre mythe individuel et mythe collectif, ce qu'elle indique dans les propos qu'elle rapporte de Christian Boltanski :

\footnotetext{
27 Ibid., p. 30.

${ }^{28}$ Voir Nadine Müller, «Adolf Wölfli's imaginäre Biographie - eine 'Individuelle Mythologie'», Mythos, $n^{\circ} 1$. Mythen in der Kunst, Würzburg, Wissenschaft und Kultur, Königshausen und Neumann, p. 77-84.

${ }^{29}$ Fabien Faure, Étienne-Martin, Mario Merz : des Demeures et des Igloos, primitivisme et dimension anthropologique de la sculpture contemporaine, L'Harmattan, 2000, p. 112, et Harald Szeemann, « Mythologies individuelles », art. cit., p. 30.
} 
«[il] a insisté sur le fait que ses photographies et ses petits objets ne racontaient pas son enfance mais celle de tout le monde: "Le passage du personnel au collectif m'a toujours intéressé. Même alors que je faisais semblant de parler de mes souvenirs, je puisais, non dans ma mémoire, mais dans le souvenir collectif." $30 »$

Mais d'après Catherine Millet, ces artistes réagissent aussi aux artistes de la génération qui les précède tout juste, les peintres de la figuration narrative qui ont marqué le paysage français en 1964 par leur grande exposition au Musée d'art moderne de la Ville de Paris, Mythologies quotidiennes ${ }^{31}$. Contrairement à ces peintres figuratifs, elle considère que "Christian Boltanski et Annette Messager ne dénoncent pas le leurre publicitaire au nom d'une vérité humaine ou politique. Leurs œuvres compromettent trop délicatement notre bon goût avec notre mauvais goût, notre besoin légitime d'idéal avec les stéréotypes culturels ». Ces stéréotypes culturels, quels sont-ils ? Millet rappelle que "[les] romans populaires dont Jean Le Gac s'inspire pour raconter les aventures de son héros sont les livres qui nous ont fait fantasmer quand nous étions enfants » et que pour sa part, Sophie Calle aussi «joue le jeu » du stéréotype, tout comme Annette Messager qui présente en 1976 ses albums de collectionneuse, parsemés de clichés populaires. Ce glissement progressif vers la mythologie non plus seulement individuelle mais aussi moderne, quotidienne, est aussi là ce qui semble distinguer deux courants possibles des mythologies individuelles. Millet n'évacue pas la question, au contraire, elle en fait un élément révélateur de la nécessité d'une croyance en un "mythe », quels que soient sa forme, son origine, rendant aussi possible son ancrage multiple. Elle pose clairement cette dichotomie qui, loin d'être un obstacle, témoigne tout simplement de la consubstantielle multiplicité de ce que peut être une mythologie de l'individu, du particulier, dans une société qui menace d'uniformisation par le stéréotype :

« On peut entendre l'expression mythologies individuelles dans le sens où celles-ci opposeraient des images privées aux images

\footnotetext{
${ }^{30}$ Catherine Millet, L'Art contemporain en France, Flammarion, 1994, p. 202.

31 Plus que le catalogue d'exposition édité à l'occasion de cette première exposition, voir surtout Gérald Gassiot-Talabot, La Figuration narrative dans l'art contemporain, cat. exp. du 1er au 29 octobre 1965, Galerie Creuze, Paris, tiré à part de la revue Quadrum [n 18,1965$]$, Bruxelles, n. p., 1965, et ensuite le second volet, en 1977, Mythologies quotidiennes 2, cat. exp. du 28 avril au 5 juin 1977, ARC 2, Musée d'art moderne de la Ville de Paris, commissariat de Gérald Gassiot-Talabot, JeanLouis Pradel, Bernard Rancillac et Hervé Télémaque, Paris, Musée d'art moderne de la Ville de Paris, 1977, n. p.
} 
médiatiques des mythologies quotidiennes. Toutefois, le mot mythologie confirme qu'il s'agit encore d'un ensemble d'idées et de croyances que, par définition, une communauté partage. ${ }^{32} »$

Millet souligne également que ce « repli sur soi » doublé parfois de gestes agressifs envers le public a eu tendance à les faire « assimiler à un comportement pathologique». Ce revers en effet troublant a conduit à s'interroger sur le basculement de la pratique artistique vers la schizophrénie, une limite que Boltanski évoque sans ambages quand il revient sur la période qui a tout juste précédé son entrée en art dans les années 1968. Dans ce débat entre folie, art brut et mythologie individuelle, c'est le conservateur et coorganisateur avec Szeemann de la documenta, Johannes Cladders, qui apporte la réponse la plus claire. Il rappelle lors d'une interview avec Jef Cornelis que l'artiste se distingue de l'aliéné par sa capacité à ne pas se laisser envahir par la névrose et à garder une distance critique :

"Je crois que cette exposition rend particulièrement explicite le fait que tous ces mondes d'images parallèles, comme ils sont appelés ici dans cette exposition, ces mondes artistiques, [...] diffèrent tous à leur manière de l'art. Et c'est évident chez le malade mental. À coup sûr, le malade mental suit quelque chose comme le chemin d'une mythologie individuelle. Ils sont possédés, et c'est la raison pour laquelle ils ne sont pas libres. C'est très clair quand on voit leurs travaux. Ils sont compulsifs. L'artiste malade mental ne peut se libérer de lui-même. Si l'on revient à Broodthaers [...], il est très fort quand il s'agit de se libérer. Il est capable de se moquer de lui-même ou de se ridiculiser. Et c'est précisément cette liberté qui manque aux autres. $^{33}$ »

La reprise du terme associé initialement aux sculptures d'ÉtienneMartin, fortement empreintes de mythologie psychanalytique, prêtait selon Szeemann à confusion, encore plus si l'on tenait le langage des artistes pour universel. À l'origine, grand mythe rejoint donc mythe personnel : de ces scories persistent dans la version de 1972, et la présence par exemple du Théâtre d'orgies et de mystères de l'actionniste viennois Hermann Nitsch a contribué au flou autour de ce qu'une mythologie individuelle pouvait avoir de collectif, d'universel ou même de sectaire. Szeemann confesse par ailleurs en 1984, à l'occasion d'une exposition d'Étienne-Martin au Centre

32 Ibid., p. 197.

33 Documenta 5, Kassel, 1972, [1972] réal. Jef Cornelis, DVD, 53', Paris Art View-Presses du réel, 2012. 
Pompidou : "cela reste très mystérieux pour [lui] », signalant que l'expression «mythologie individuelle » telle qu'elle fut interprétée dans le monde de l'art avait sans doute dépassé son intention première. Dans ce texte, il distingue la notion spécifique de «mythologie individuelle » appliquée à Étienne-Martin et celle, plus générale, utilisée pour la documenta, qui associait dans le pavillon des mythologies individuelles une pluralité de singularités artistiques. Chez Étienne-Martin, la demeure reste associée à une représentation archétypale de la maternité qui se confond avec le personnage mythique de Parsifal. Le héros doit retrouver sa propre histoire et reconstituer ainsi sa véritable identité :

«Pour comprendre ces sculptures, il faut avoir présente cette vérité, le mystère de la croissance et la notion de "mythologie individuelle" d'Étienne-Martin basée sur l'interrelation du mythe individuel avec le mythe collectif. Le départ du giron de la mère - archétype de la Demeure - génère la nostalgie de cette caverne protectrice dans la vie à vivre, dans le monde extérieur. L'espace vital est organisé selon l'idée directrice de la "demeure" initiale, les épreuves infligées ramenées à la mémoire d'elle. Ce Parsifal, jeune ingénu parce qu'il ne savait pas qui était sa mère, doit devenir le Parsifal qui connaît sa mère et son origine. ${ }^{34}$ »

D'après Szeemann, ces brumeuses mythologies individuelles qui n'ont en apparence pas de dénominateur commun sont "parties d'une notion d'une histoire de l'art de l'intensité » et procèdent de «l'identité sensible de l'intention et de l'expression»35. D'une certaine manière, les mythologies individuelles, dont les frontières restent indistinctes, avaient surtout le grand avantage d'abolir dans le champ critique l'opposition entre « art conceptuel et réalisme ». En effet pour Szeemann, d'une part, chaque artiste est un «mythologue individuel », qu'il soit conceptuel, structuraliste ou réaliste : d'autre part, peu importe les caractéristiques formelles des productions puisqu'il s'agit précisément de faire cohabiter des singularités dans le cadre d'une catégorie " parfaitement ouverte».

C'est à cet endroit que Szeemann fait le lien le plus explicite entre «mythologies individuelles » et art brut, ou, plus largement, un art

${ }^{34}$ Harald Szeemann, "Cela reste très mystérieux pour moi », Étienne-Martin, Les Demeures, cat. exp. du 19 avril au 11 juin, 1984, Paris, Centre Georges-Pompidou, p.12. Il s'agit d'un texte différent de celui que l'on peut trouver dans l'autre ouvrage sur Étienne-Martin publié en 1991 chez Adam Biro, «L'extérieur décide de la mesure, l'intérieur détermine la forme », dans lequel Szeemann revient sur la différence avec la documenta 5 en incluant des extraits de son texte paru dans Kunstnachrichten en 1972.

35 Harald Szeemann, « Mythologies individuelles », art. cit., p. 30-31. 
populaire qui se conçoit en dehors des «belles et bonnes» formes attestées de l'art. Szeemann cite librement une série d'œuvres et thèmes de son exposition: "L'Égalité Espace-Événement de Paul Thek; L'archéologie devenue forme "égocentrique" de Christian Boltanski; Le "mysticisme hiérarchique" (Ammann) d'ÉtienneMartin comme expression religieuse ${ }^{36}$ ", et poursuit jusqu'aux "séries picturales des malades mentaux». Il donne encore pour exemple « la foi naïve d'Auguste Herbin [...], la quête obsessionnelle du Viennois Nitsch, de Brus [...] : la diversité de la représentation du soi, l'intensité dans la permanence avec laquelle Beuys transmet aux visiteurs sont idées de la démocratie $[\ldots]^{37} »$. Mis sur le même plan et dans une forme de progression en intensité, cette proximité entre artistes aliénés et du marché se révèle aussi productive pour ces derniers, car ils vont eux aussi utiliser, comme Annette Messager, ces « répertoires de formes » pour les réinvestir dans leurs œuvres.

Convaincu que «l'art des années 1970 s'orientera vers l'intériorité, et c'est ce que souligne tout un bâtiment, la Neue Galerie ", Szeemann sent distinctement l'air du temps. Il pousse toutefois les mythologies individuelles dans une dimension propre à une philosophie New Age. Cette philosophie holistique a donné lieu à un véritable courant de développement personnel d'où la dimension mystique n'était pas du tout exclue ${ }^{38}$. On reconnaît les accointances de ce discours qui renvoie à une cosmogonie artistique et ferait communiquer l'intériorité avec un plus grand ordre, comme une «tentative de chacun d'opposer son propre ordre au grand désordre ». Szeemann déclare dans cet ordre d'idée que "les "Mythologies individuelles" tentent de donner à la " $d 5$ " la dimension d'un espace métaphysique dans lequel chacun pose les signes et signaux exprimant son monde personnel». Il poursuit son raisonnement en incluant l'art des fous, l'art brut, dans cette vaste entreprise humaniste en concluant qu' «il est difficile de désigner un fou comme fou lorsque, comme à Cassel, on a appris à apprécier ces mythologies individuelles comme expressions existentielles des valeurs de notre voisin ${ }^{39}$ »- voisinage de la folie d'autant plus concret que les tableaux-dessins de malades mentaux et l'art religieux populaire étaient exposés à proximité de la section.

\footnotetext{
$36_{\text {Ibid., p. } 31 .}$

37 Idem.

${ }^{38} \mathrm{Peu}$ connu en France, Joseph Campbell aux États-Unis occupait la chaire de mythologie comparée à l'Université Sarah Lawrence. Il fut surtout le théoricien de l'usage de la mythologie dans le développement personnel et l'auteur du célèbre Puissance du mythe (Power of Myth, 1991) ou encore Des mythes pour se construire (Myths to live by, 1972), un recueil de conférences données de 1958 à 1971.

${ }^{39}$ Harald Szeemann, « Mythologies individuelles », art. cit., p. 32.
} 


\section{Dépasser la folie, s'emparer du quotidien}

Avec l'idée d'un musée des obsessions, un pli psycho-mythique aux accents plus germaniques semble toutefois résolument pris. Les références à Schreber et son Denkwürdigkeiten eines Nervenkranken (1903), à Jung et ses ouvrages Gestaltung des Unbewussten (1950), Bewusstes und Unbewusstes (1957) ou à son Archetypen und die kollektive Unbewusste, mais aussi au Vocabulaire de la psychanalyse de Laplanche et Pontalis, et enfin à la schizo-analyse de Deleuze et Guattari dans L'Anti-OEdipe, Szeemann bascule dans un musée de l'obsession similaire à celui que Charles Mauron avait esquissé pour la littérature. On voit là clairement le croisement entre le mythe personnel du névrosé décrit par Lacan en 1953 et la «mythologie individuelle» soutenue par l'énergie vitale qu'est l'obsession, «présentée plus ou moins intensément». Elle nécessite le dépassement de la «biographie, qui fut dominante dans la documenta 5 » afin d'atteindre la «libération de l'énergie démente qui, à nouveau, veut créer, créera un tout 40 ». Force est de constater que Szeemann est, dans cette perspective, bien loin de la déconstruction des mythologies petites-bourgeoises de Roland Barthes.

Malgré ce discours très ésotérique de Szeemann, qui lie très nettement l'art brut, l'art des fous, l'obsession, la névrose et la mythologie individuelle, cette dernière a cependant continué une vie propre sous une autre forme, moins psycho-chargée, voire «apsychanalytique » et bien plus culturelle. Beaucoup d'idées émergent de ces courts textes de Szeemann, toutes n'allant pas dans la même direction. Szeemann entre notamment dans son "Musée des obsessions » à partir de la mythologie individuelle, la laissant derrière lui, comme pour se débarrasser d'une notion trop encombrante ou comme si elle ne pouvait avoir de vie ultérieure, artistique ou collective ${ }^{41}$. Pourtant, certains de ses propos renvoient indirectement à sa conception d'une mythologie dont l'individu se serait emparé, un individuum qu'il projette dans le futur, et qui semble préfigurer un programme culturel commun. Un commentaire du critique Dieter Bachman face à la Dokumentation über A.S. [l'artiste brut Armand Schulthess] de Ingeborg Lüscher, la compagne de Szeemann, va dans ce sens ${ }^{42}$. Il induit la possibilité d'une réappropriation par la liberté artistique de la mythologie individuelle, idée que Szeemann fait sienne. Bachman interprète

\footnotetext{
40 Harald Szeemann, « Agence pour le travail intellectuel... », art. cit, p. 49.

41 Harald Szeemann, "La réflexion sur "Documenta" et surtout sur les "Mythologies individuelles" conduisit à l'idée d'un "Musée des obsessions" », ibid., p. 51.

42 Ingeborg Lüscher, Dokumentation über A.S. Der grösste Vogel kann nicht fliegen, Dumont Schauberg, Cologne, 1972.
} 
cette documentation sur l'artiste brut du Tessin comme un «appel, adressé à chacun en particulier, à justement réaliser sa propre mythologie individuelle ». Reprenant à son compte l'idée de l'individu artiste qui permet à chacun d'être créatif, il considère que la «documenta 5 qui semble élitaire, réveille cette pensée démocratique ${ }^{43}$ », rendant possible la dissémination de la mythologie individuelle au-delà du monde de l'art.

En 2006, Catherine Millet réarticule cette idée pour la renverser dans une posture critique propre à une culture française paradoxalement baignée de matérialisme marxiste et d'affirmation de l'individualité. D'après elle en effet, la génération d'artistes post1968 se fait le catalyseur-auteur de productions qui «ne fondent plus les croyances de la communauté [mais] expriment les obsessions des individus ${ }^{4}$ ». Mais comme elle le fait remarquer plus loin, si Boltanski incarne aussi bien cette pratique élargie des «mythologies individuelles", c'est parce qu'il recycle et met en œuvre les composantes scénographiques des mythologies quotidiennes modernes décrites par Roland Barthes, Guy Debord, Pierre Bourdieu, mais aussi par son propre son frère, Luc Boltanski. À cet égard, l'intégration de la photographie a permis d'hybrider la mythologie individuelle szeemannienne, proche de l'Art brut, avec d'autres acceptions modernes de la notion de "mythologies », au pluriel, la rendant plus proche des images façonnant le quotidien. Les mythologies individuelles marquent aussi très fortement le lien qui unit l'art à la vie, la documenta étant elle-même «la vie concentrée sous forme d'exposition », comme le déclare Szeemann ${ }^{45}$. La pluralité des médias présents à la documenta de 1972 a fait éclater le concept de mythologie pour le transposer dans le monde des images (« Imageries d'aujourd'hui » était le sous-titre de la documenta) et du contemporain, un monde plongé dans les processus mythographiques médiatiques, désormais augmenté d'une part esthétique et fictionnalisante nouvelle.

L'influence directe de l'art brut fut rapidement revendiquée chez des artistes contemporains comme Jean Tinguely ou Niki de Saint Phalle qui découvrent le Facteur Cheval en 1962. Niki de Saint Phalle reconnaît que le bricoleur de génie a radicalement modifié sa pratique artistique: "Ces œuvres m’ont profondément inspirée, alors qu'elles n'étaient pas considérées comme sérieuses par les

\footnotetext{
43 Harald Szeemann, « Mythologies individuelles », art. cit., p. 32.

${ }^{44}$ Catherine Millet, L'Art contemporain. Histoire et géographie, Champs Flammarion, 2006, p. 90.

${ }^{45}$ Lucia Pesapane, «Introduction», op. cit., p. 95. Nombre d'artistes de la documenta 5 ont été présentés à nouveau lors de l'exposition Hors limites, l'art et la vie 19521994, cat. exp., Centre Georges-Pompidou, 1994, qui avait aussi pour vocation de placer la France au cœur de cette tendance esthétique et culturelle.
} 
autres artistes qui les voyaient comme du "Folk Art". 46 » De leur côté, les œuvres de Jean Dubuffet témoignent d'emprunts formels évidents aux artistes bruts qu'il défendait par ailleurs. Toutefois, cette influence prend une tout autre dimension chez les artistes des mythologies individuelles qui associent directement l'art brut à la pratique d'une esthétique contemporaine ancrée dans le quotidien.

Dans son livre-entretien La Vie possible de Christian Boltanski, réalisé comme une psychanalyse avec Catherine Grenier, Christian Boltanski revient sur ses débuts d'artiste, son enfance, et fournit de précieuses indications sur la genèse de ses œuvres. Il raconte notamment comment il a pu rejoindre la galerie de Ileana Sonnabend en présentant sa série des Petits couteaux, ses boîtes de biscuits ou encore les boulettes de terre qu'il réalisait méthodiquement et compulsivement pour tenter d'obtenir une sphère parfaite ${ }^{47}$. Boltanski se défend pourtant d'avoir jamais été dans l'art brut: «Non, je n'étais pas dans l'art brut, vraiment pas. Il y a vaguement l'idée de la relique dans mon travail, mais il n'y avait pas du tout l'idée de magie. Le projet était de garder des traces, de lutter contre la mort... ${ }^{4}$ », et Catherine Grenier suggère que cette distance est marquée par un second degré présent dans les œuvres de Boltanski. L'art brut intègre en fait, par une mise en abyme cocasse, la mythologie individuelle de l'artiste : ses pratiques compulsionnelles, même contrôlées, participent très nettement des caractéristiques de cette tendance, d'autant qu'il admet lui-même avoir eu des comportements schizophréniques, voire «un retard mental certain, de cinq ans ou plus» ou encore avoir été "violent contre [lui]même ".

Les Archives Christian Boltanski, compilées par Robert Calle, l'un de ses premiers collectionneurs, en disent pourtant long sur les accointances formelles entre les œuvres de jeunesse de Boltanski et l'art brut: ses centaines de peintures expressionnistes, presque toutes détruites, ses mannequins géants, ses constructions en sucre, en pomme, d'une facture de mauvaise qualité et à l'esthétique amateur, son air volontairement idiot dans les Saynètes comiques, créent une véritable proximité entre les œuvres de Boltanski et l'imagerie d'Épinal des productions brutes. La description que fait Bob Calle de ses débuts renvoie nettement aux pratiques artistiques populaires incluant notamment des images de piété :

\footnotetext{
${ }_{46}$ Cité par Lucienne Peiry, L'Art brut, Tout l'art, Flammarion, 1997, p. 239.

47 « Moi chaque jour, je fais des boules de terre, des milliers de boules de terre, et jamais je n'arriverai à modeler des boules parfaitement rondes. » Christian Boltanski et Catherine Grenier, La Vie possible de Christian Boltanski, Fiction et Cie, Seuil, ${ }^{48}$ Ibid. 2010, p. 49
} 
«[...] de 1955 à 1969, Christian Boltanski, avant d'entreprendre ce qu'il appelle sa réelle activité artistique, peint sur du contreplaqué des sujets religieux ou historiques, puis, vers 1968, des événements dramatiques individuels. [...] Dans le même temps, de 1963 à 1970, Christian Boltanski réalise des mannequins grandeur nature ou grandes poupées. ${ }^{49}$ »

Ces informations recoupent également un autre témoignage de Boltanski qui ajoute que beaucoup de ses productions, à l'origine, n'étaient pas vraiment de l'art, et tout du moins n'étaient pas destinées au monde de l'art: "À l'âge de 20 ans, par exemple, j'avais construit une grande ville en sucre - mais ce n'était pas une œuvre artistique - avec des milliers de morceaux de sucre. J'avais taillé et assemblé ces morceaux de sucre en forme de maisons et puis, j'en ai eu marre, j'y ai mis le feu. ${ }^{50}$ » Une autre expérience similaire fut réalisée avec ses deux frères, Jean-Élie et Luc, mais en utilisant des pommes : au fil des jours, le centre ville s'assombrissait sous l'effet de l'oxydation, tandis que la banlieue était d'une neuve fraîcheur. Bien des années après et par un effet rétrospectif, on imagine parfaitement que, de son point de vue, cet épisode " art brut» a été entièrement intégré et métabolisé par son activité artistique ultérieure. Boltanski commente sa rencontre avec l'art brut dans le processus de distanciation critique avec sa propre pratique :

"Annette [Messager] m'a alors apporté une chose très importante: l'Art brut, que j'avais un peu vu mais pas vraiment. Elle avait les Cahiers de l'Art brut, qu'elle m'a montrés, et je pense que ça a eu une grande influence sur moi. Par rapport à cette idée de répétition, d'objets apparemment inutiles et fragiles... Ce qui est beau dans l'Art brut, c'est que tu as un petit dessin sur un bout de papier minable et qu'en même temps, c'est la cosmogonie, Dieu. Il y a une grande différence entre ce qui est représenté et la pauvreté du moyen. J'avais commencé ce travail avant de connaître Annette et avant de connaître l'Art brut, mais cela m'a renforcé dans l'idée. ${ }^{51}$ »

Ce fort ancrage dans l'univers de l'art brut vient d'Annette Messager, originaire du Pas-de-Calais, alors étudiante aux arts décoratifs et qui a une conscience plus nette de la radicalité à la fois formelle et symbolique de ces œuvres populaires et singulières. Elle

${ }^{49}$ Robert Calle, Archives Christian Boltanski 1, éd. 591, 2009, p. 13.

${ }^{50}$ Christian Boltanski et Catherine Grenier, La Vie possible de Christian Boltanski, op. cit.,

$51_{\text {Ibid., p. } 49 .} 4$. 
reconnaît aussi une force authentique à l'œuvre chez ces artistes et se les réapproprie dans sa propre activité mytho-poétique. Mais l'art brut est aussi pour Annette Messager un souvenir d'enfance qui a tapissé l'arrière-plan de sa production personnelle. Dans sa ville natale, Berck, comme elle le raconte, «tout le monde faisait de l'art ${ }^{52}$ », et elle est initiée d'abord avec son propre père à l'art religieux populaire, à la photographie qu'il pratiquait en amateur, et à l'art brut, puisqu'il lisait les écrits de Dubuffet. Plus tard, comme Catherine Grenier le rapporte au sujet de ses débuts en art, elle s'intéresse à la simplicité des matériaux employés et à leur proximité familière. Ainsi, les broderies de Jeanne Tripier, découvertes en 1967 lors de l'exposition Art Brut de Jean Dubuffet au Musée des arts décoratifs, rencontre son intérêt pour «les petits objets» qu'elle attribue par ailleurs à l'influence des surréalistes et en particulier des photographies dans Nadja. Elle partage en effet leur goût pour « les expressions singulières de l'art », et alors que la période est plutôt défavorable à cet esprit post-dada, elle se positionne nettement à contre-courant de l'engouement pour l'art conceptuel, le minimalisme et toutes les formes esthétiques de la fin du modernisme 53 . Fidèle donc au mauvais goût hétéroclite des premiers surréalistes, elle déclare : « [j]'ai toujours beaucoup emprunté à l'art religieux et populaire, à l'Art brut, aux images de l'hystérie, mais aussi aux traditions arabes, indiennes ou tantriques... [...] L'art conceptuel m'a intéressée au même titre que l'Art brut, l'astrologie ou l'art religieux [...]». Quand elle précise "ce sont pour moi des répertoires de formes ${ }^{54}$ », la citation formelle devient limpide, notamment dans la série "Mes dessins d'enfant» (par exemple Maman téléphone, 1971-197255) qui rappellent immédiatement ceux d'Aloïse Corbaz. À partir d'images de l'enfance, d'objets simples et quotidiens, la mythologie individuelle des artistes contemporains entremêle souvenirs personnels et déconstruction des stéréotypes, pour faire entrer la fabrique de l'individu dans un bricolage mythopoétique marqué par la réflexivité critique.

\section{Conclusion : la mythopoétique contemporaine}

Les artistes des mythologies individuelles en provenance de la scène parisienne reflètent donc, et c'est un sentiment partagé par le

\footnotetext{
${ }^{52}$ Catherine Grenier, Annette Messager, Flammarion, 2000, p. 42.

53 Ibid., p. 45.

${ }^{54}$ Lucienne Peiry, L'Art brut, op. cit., p. 241-242.

55 Reproduit dans Annette Messager, Mot pour mot, Presses du réel - Violette éditions, 2006, p. 42-43.
} 
critique Günter Metken, une spécificité française ${ }^{56}$. Celle-ci hérite d'autres traditions et références que celles revendiquées par Szeemann : d'une part, celle, majeure, du livre d'artiste, et d'autre part, de l'histoire récente de la notion de mythologie. Réactualisée dans les sciences humaines et à travers l'étude des médias par Roland Barthes, elle est réappropriée par le monde de l'art avec les représentants de la figuration narrative, comme en atteste en 1964 l'exposition Mythologies quotidiennes au Musée d'art moderne de la Ville de Paris. C'est autour de la reconfiguration narrative en art, autour du montage entre récit de soi et photographie, que les mythologies individuelles vont véritablement trouver en France leur expression extériorisée et formelle, s'affranchissant dès lors de leur caractère "obsessionnel ». Dans le même temps, il ne faut pas mésestimer le rôle de la légitimation de l'autobiographie comme genre, sous la plume de Philippe Lejeune qui scelle Le Pacte autobiographique (1975), étendu deux ans plus tard, en 1977, par Serge Doubrovsky à la notion d'autofiction. Cette tendance autographique accrédite le pressentiment de Szeemann que les années 1970 ont résolument été les années du moi, fondant une culture spécifique de l'identité à travers narration et esthétique. Si les formes les plus communes sont l'album de famille, et l'on peut se référer au numéro de la défunte revue La Recherche photographique consacré à la famille ainsi qu'au livre de Bourdieu, «Un art moyen », pour délimiter les contours de ces pratiques de masse, les positions radicales des avant-gardes nous permettent de voir les formes les plus complexes et critiques de ces représentations de soi de masse. Les dispositifs hétérogènes (sculptures, dessins, photos, récits) des artistes des mythologies individuelles nous informent pour beaucoup sur la représentation de l'identité et de l'individu à ce moment charnière $\mathrm{du} \mathrm{XX}^{\mathrm{e}}$ siècle qui voit les médias (au sens de médium: télévision, vidéo, photographie) de plus en plus accessibles à la masse. Les artistes des mythologies individuelles de la scène parisienne ont donc aggloméré ces diverses tendances pour incarner un nouvel art brut mythologico-autobiographique: les documents amateurs, les pratiques compulsives, la fiction de soi (pathologique

${ }^{56}$ Christian Boltanski et Catherine Grenier, La Vie possible de Christian Boltanski, op. cit. : "Il a été un des seuls à comprendre qu'il y avait une sorte de groupe français ", p. 72. Voir également Günter Metken, Spurensicherung. Kunst als Anthropologie und Selbstforschung. Fiktive Wissenschaften in der heutigen Kunst (Conservation des traces. L'art comme anthropologie et recherche de soi. Savoirs fictifs dans l'art actuel), Cologne, Dumont Aktuell, 1977, et surtout son article "Das fruchtbare Missverständnis. Lévi-Strauss und die individuelle Mythologien (Le malentendu fructueux. Lévi-Strauss et les mythologies individuelles) », in Erika Billeter, Mythos und Ritual in der Kunst der siebzieger Jahre, cat. exp. du 7 novembre 1981 au 3 janvier 1982, Kunstverein de Hambourg, Zürich, Kunsthaus, 1981. 
chez les aliénés, jouée chez les artistes), les jeux sur les dédoublements identitaires, les névroses narcissiques, le mythe familial, tout cet intertexte participe d'un brouillage entre l'idée d'une construction identitaire qui participe d'une configuration psychique et celle d'une représentation de soi qui s'esthétise et se modèle dans l'espace public.

L'emprunt ou le prolongement d'une dimension brute des documents est pourtant trahi par l'usage de la photographie, une technique presque totalement exclue de la nomenclature établie par Dubuffet et, par conséquent, des collections d'art brut. Cette technicité réflexive serait-elle déjà une mise à distance de l'authentique expression de soi ? Sans doute, mais l'usage de la photographie chez les artistes des mythologies individuelles croisée à des emprunts formels dans le répertoire de l'art brut ou populaire apparaît précisément comme le symptôme de la distance critique qui les sépare des aliénés. La photographie les fait basculer du côté de la représentation pure, et non plus dans la simple, naïve et mythologique expressivité d'un sujet singulier. Cette distanciation est aussi un acquis des sciences humaines, Boltanski et Messager étant instruits des travaux de Bourdieu, Lévi-Strauss et Barthes. Quelle innocence avoir encore après ces positions réflexives? Cette période des années 1968-1972 est le moment charnière où le bricolage mytho-poétique acquiert le statut d'activité artistique à part entière et fait entrer l'art dans le "contemporain », un art du temps dans lequel le sujet-artiste vit, « un art pertinent au moment où il est fait» et qui ne peut plus être considéré comme de l'art conceptuel, pour reprendre les termes de Laurence Weiner lors de la documenta $5^{57}$. Et en intégrant Miroslav Tichý, seul photographe associé à l'art brut, dans l'une de ses dernières expositions en 2004, la Biennale de Séville, Szeemann semble avoir lui-même jeté le dernier pont qui maintenait le territoire de l'art brut à l'état insulaire pour le faire entrer pleinement dans la contemporanéité58.

On assiste avec l'entrée dans le contemporain, et précisément l'art contemporain, à une déconstruction des catégories, caractéristique de l'ère postmoderne, art brut et art conceptuel étant assimilés dans un processus global qui implique l'identité même de l'individu. Cette intégration totale de l'artiste et de son identité procède d'un engagement propre aux artistes de l'art brut, mais elle est prolongée et replacée dans un système artistique légitimé et institutionnel. En héritant de cette première génération d'artistes des mythologies

57 Documenta 5, Cassel, 1972 [1972], réal. Jef Cornelis, op. cit.

58 Voir à ce sujet Marc Lenot, "L'invention de Miroslav Tichý », Politique des images / illustrations photographiques, Études photographiques, $\mathrm{n}^{\circ} 23$, mai 2009, p. 216238. 
individuelles à la fois post-bruts et post-conceptuels, la construction du mythe personnel est devenue une affaire esthétique et de représentation de soi à part entière. Ce changement de paradigme a ouvert en grand la porte à des artistes comme Sophie Calle, qui ont fondé la quasi-intégralité de leur œuvre sur ce principe. Et ce type d'œuvre, résolument contemporaine, fait elle-même écho au mouvement de masse que le tournant des $\mathrm{XX}^{\mathrm{e}}$ et $\mathrm{XXI}^{\mathrm{e}}$ siècles a vu émerger, celui des mythologies diaphanes de l'individu à l'ère numérique, un individu aux représentations dématérialisées et dépouillées de toute trace de cette expressivité brute qui incarnait pourtant la marque même de la singularité. 